\title{
Quiste periodontal lateral, reporte de un caso
}

Lateral periodontal crst, case report

Cisto periodontal lateral, relato de caso clínico

\section{Mariana Celeste Villalobos Medina ${ }^{1}$ Jesús Miguel Jiménez Matute ${ }^{2}$}

Recibido: 29 de enero de 2018 Aprobado: 9 de marzo de 2018

Publicado: 20 de marzo de 2019

Cómo citar este artículo: Villalobos-Medina M, Jiménez- Matute JM. Quiste periodontal lateral, reporte de un caso. Rev Nac Odontol. 2019;5(28):1-10. doi: https://doi.org/10.16925/2357-4607.2019.01.08

Reporte de caso. https://doi.org/10.16925/2357-4607.2019.01.08

1 Universidad Central de Venezuela (UCV), Caracas, Venezuela ORCID: https://orcid.org/0000-0003-1188-9215

2 Universidad Central de Venezuela (Ucv), Caracas, Venezuela ORCID: https://orcid.org/0000-0003-3496-2390

Correo electrónico: jmjma1@hotmail.com 


\section{Resumen}

Introducción: el Quiste Periodontal Lateral (QPL) también denominado quiste botrioide odontogénico, es considerado un quiste odontogénico del desarrollo, poco frecuente, de mayor incidencia entre la quinta y séptima década, con predilección por el sexo masculino. Esta lesión es generalmente asintomática y radiográficamente se presenta como una imagen radiolúcida con bordes bien definidos de forma redonda u ovoide.

Presentación del caso: en el presente artículo se hace referencia a un caso clínico soportado con la revisión de la literatura, de una lesión ubicada entre el incisivo lateral y el canino inferior derecho en un paciente masculino de 37 años de edad. Se le realizó radiografía panorámica y periapical, por presentar al examen clínico una lesión blanquecina a nivel del vestíbulo bucal en la zona anteroinferior derecha, de aproximadamente $8 \mathrm{~mm}$ de diámetro, blanda a la palpación de base sésil. Radiográficamente se detectó una imagen radiolúcida ubicada lateralmente entre el 42 y 43 . La lesión fue eliminada quirúrgicamente en su totalidad sin la aplicación posterior de material regenerador óseo, se tomó biopsia excisional de la lesión y se corroboró el diagnóstico presuntivo.

Conclusión: la extirpación de la lesión en un solo tiempo quirúrgico constituye un tratamiento satisfactorio para este tipo de patologías.

Palabras clave: quiste periodontal lateral, patología, quiste odontogénico, quiste botrioide odontogénico.

\section{Summary}

Introduction: Lateral Periodontal Cyst (CPL), also called botrioid odontogenic cyst, is considered an uncommon odontogenic cyst of development, with a higher occurrence between the fifth and seventh decade, predominantly among males. This lesion is generally asymptomatic and radiologically presents as a radiolucent image with well-defined round or oval borders.

Case report: In this article, we refer to a clinical case, supported by literature review, of a localized lesion between the lateral incisor and the right lower canine in a male patient of 37 years. A panoramic and periapical radiograph was performed, because the clinical exam presented a whitish lesion at the level of the buccal vestibule in the right anteroinferior area, approximately $8 \mathrm{~mm}$ in diameter, soft to palpation of the sessile base. Radiographically, a radiolucent image located laterally between 42 and 43 was detected. The lesion was surgically removed in its entirety without the subsequent application of bone regenerating material; an excisional biopsy of the lesion was performed and the presumptive diagnosis was corroborated.

Conclusion: the elimination of the lesion in a single surgical time was a satisfactory treatment for this type of pathologies.

Keywords: periodontal cyst, pathology, odontogenic cyst, odontogenic cyst botrioid.

\section{Resumo}

Introdução: o Cisto Periodontal Lateral (CPL) também denominado cisto odontogênico botrioide, é considerado um cisto odontogênico do desenvolvimento pouco frequente, de maior ocorrência entre a quinta e sétima década, com predominância entre o sexo masculino. Essa lesão é geralmente assintomática e radiograficamente se apresenta como uma imagem radiolúcida com bordas bem-definidas de forma redonda ou oval.

Apresentação do caso: neste artigo, faz-se referência a um caso clínico, apoiado com a revisão da literatura, de uma lesão localizada entre o incisivo lateral e o canino inferior direito em um paciente masculino de 37 anos. Foi realizada radiografia panorâmica e periapical, por apresentar no exame clínico uma lesão esbranquiçada no nível do vestíbulo bucal na área anteroinferior direita, de aproximadamente $8 \mathrm{~mm}$ de diâmetro, mole à palpação de base séssil. Radiograficamente foi detectada uma imagem radiolúcida localizada lateralmente 
entre o 42 e 43. A lesão foi eliminada cirurgicamente em sua totalidade sem a aplicação posterior de material regenerador ósseo; foi tomada biópsia excisional da lesão e foi corroborado o diagnóstico presuntivo.

Conclusão: a eliminação da lesão em um só tempo cirúrgico constituiu um tratamento satisfatório para esse tipo de patologias.

Palavras-chave: cisto periodontal lateral, patologia, cisto odontogênico, cisto odontogênico botrioide.

\section{Introducción}

Los quistes odontogénicos son clasificados por la Organización Mundial de la Salud como inflamatorios y de desarrollo según su revestimiento epitelial. Los quistes periodontales laterales (QPL) son considerados quistes del desarrollo o de origen incierto $(1,2)$.

Los QPL se definen como quistes de desarrollo no inflamatorios localizados adyacentes o laterales a la raíz de un diente vital. La ubicación más frecuente de este quiste está a nivel de los premolares mandibulares, pero se ha informado que ocurre en las otras áreas (3).

La etiología del quiste periodontal lateral no está clara, se cree que la lesión se origina a partir de restos epiteliales odontogénicos - restos de Serres-, aunque existe controversia con respecto a la posible implicación de restos de lámina dental, restos de Malassez o el epitelio del esmalte reducido que podrían causar la formación y el desarrollo del QPL (4-7).

El QPL es más prevalente en adultos entre la quinta a séptima década, con una edad media de 52 años, sin preferencia por raza o sexo. La localización de QPL notificada con mayor frecuencia es el área premolar mandibular, seguida de la región anterior del maxilar (3, 7-10).

El quiste periodontal lateral es uno de los quistes de menor incidencia entre los quistes odontogénicos del desarrollo. Dado que los pacientes con QPL generalmente son asintomáticos y rara vez se ha informado de dolor u otros síntomas clínicos, la lesión a menudo se descubre en un examen radiográfico de rutina. Las radiografías del quiste periodontal lateral muestran un área radiolúcida redonda u ovoide bien circunscrita, generalmente con un margen esclerótico. La mayoría de ellos tienen menos de $1 \mathrm{~cm}$ de diámetro. Las características radiográficas de QPL no son patognomónicas y pueden parecerse a un queratoquiste odontogénico o un quiste radicular lateral $(3,9,11,12)$.

Histológicamente el QPL se compone de una cavidad quística con una pared de tejido conectivo con revestimiento epitelial escamoso no queratinizado de 1-5 células de grosor que se asemeja al epitelio reducido del esmalte. El revestimiento epitelial presenta engrosamientos o placas focales, en los que a menudo se han encontrado células epiteliales claras que contienen glucógeno. El tejido conjuntivo subyacente al epitelio exhibe una zona de hialinización $(11,13)$. 
Los quistes odontogénicos no tratados pueden causar reabsorción de la raíz, desplazamiento de los dientes, expansión y dolor. El tratamiento de QPL incluye la extirpación quirúrgica de la lesión mediante enucleación conservadora y el seguimiento radiográfico del paciente para controlar la recurrencia. Durante el período de cicatrización de 6 meses a 1 año, la regeneración ósea ocurrirá dentro del defecto óseo y las recurrencias son poco comunes. Por otro lado, se han utilizado diferentes enfoques regenerativos, incluida la técnica de regeneración tisular guiada (GTR) combinada con aloinjerto óseo descalcificado liofilizado (DFDBA) y plasma rico en plaquetas (PRP) en el tratamiento intraóseo de cavidades quísticas (14-16).

\section{Presentacion Del Caso Clinico}

Paciente masculino de 37 años de edad, natural y procedente de Caracas, Venezuela, se presenta a consulta de manera asintomática refiriendo movilidad del incisivo lateral derecho inferior desde hace aproximadamente 6 meses y presencia de lesión blanquecina a nivel del vestíbulo bucal en la zona anteroinferior derecha.

Al examen clínico intrabucal se observa una lesión de coloración blanquecina, de aproximadamente $8 \mathrm{~mm}$ de diámetro, asintomática a la palpación, de base sésil y consistencia blanda. A la prueba de vitalidad pulpar se presentó vital el incisivo y canino inferior derecho a pesar de observar movilidad grado III y ligero desplazamiento en el incisivo lateral de la zona mencionada (figura 1).

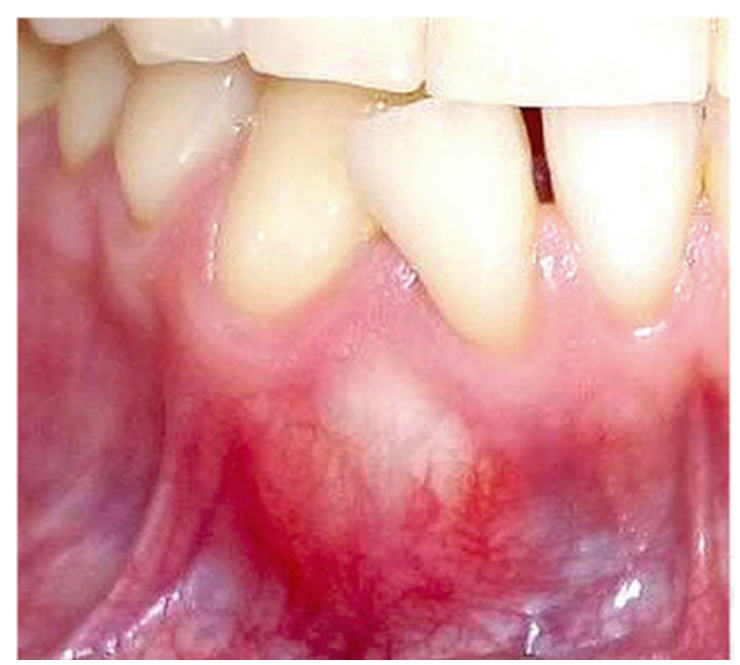

Figura 1. Aspecto clínico intra bucal.

Fuente: elaboración propia 
Radiográficamente se detectó una imagen radiolúcida ubicada lateralmente entre el 42 y 43 motivo por el cual se le indicó una tomografía axial computarizada para evaluar la extensión de la lesión, evidenciándosE una imagen radiolúcida de aproximadamente $1 \mathrm{~cm}$ de diámetro, con bordes circunscritos de forma ovoide y desplazamiento lateral de las raíces del 42 y 43 (figuras 2 y 3 ).
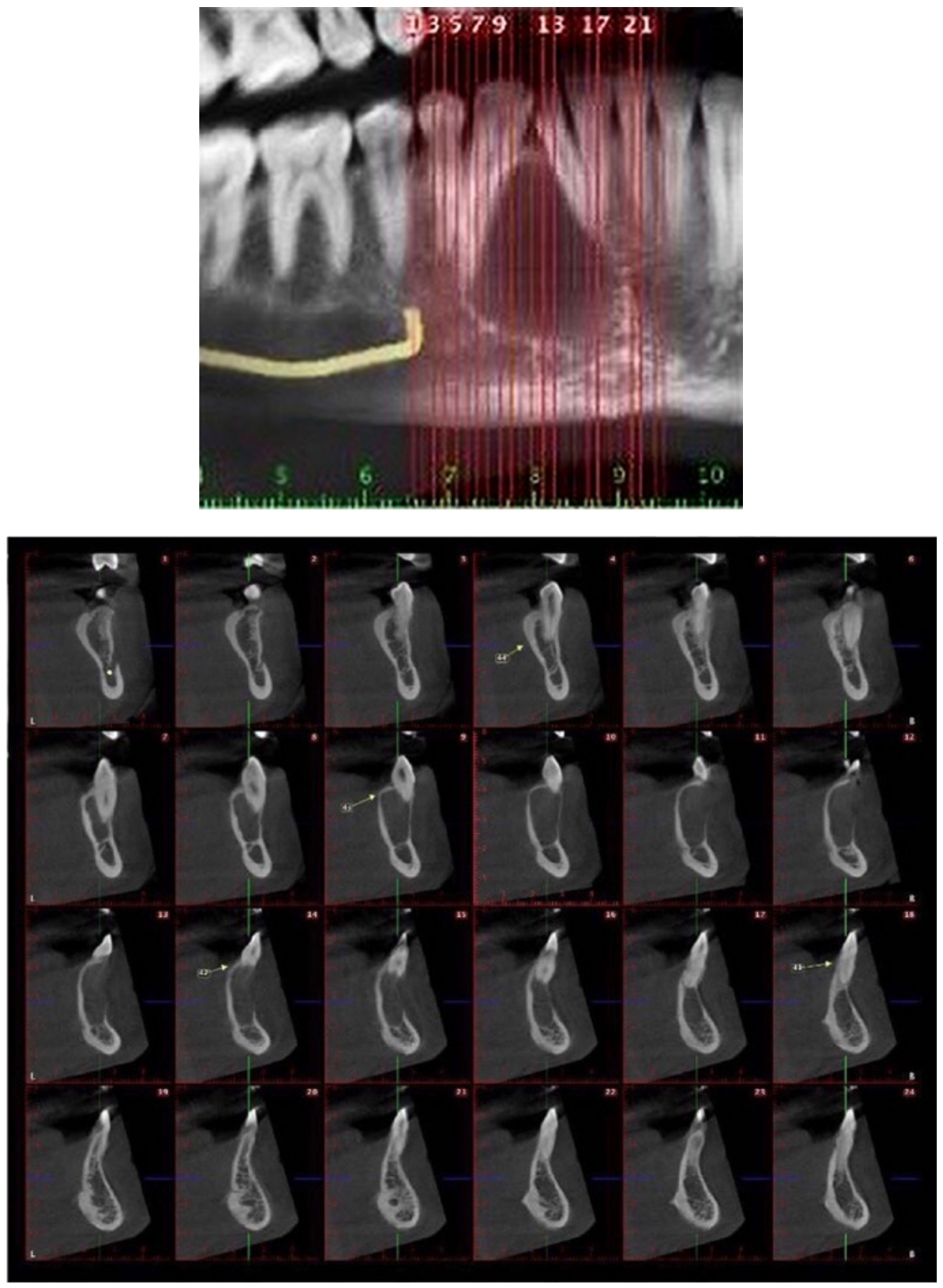

Figuras 2 y 3. Tomografía Axial Computarizada de la zona 42-43. Fuente: elaboración propia 
Con base en el examen clínico extrabucal e intrabucal se determinaron los siguientes diagnósticos diferenciales: quiste periodontal lateral, queratoquiste odontogénico y quiste radicular lateral.

Se indicaron los exámenes complementarios para posteriormente proceder al tratamiento definitivo, el cual se inició con la anestesia troncular del nervio dentario inferior e infiltrativa del mentoniano en la zona antero inferior del proceso mandibular, luego se procedió a realizar una incisión intercrevicular de forma Newman completa con dos liberaciones verticales, una en mesial del 44 y otra hacia distal del 41 . Se levantó el colgajo mucoperióstico de espesor completo para exponer la lesión y el tejido óseo circundante de la misma (figura 4).

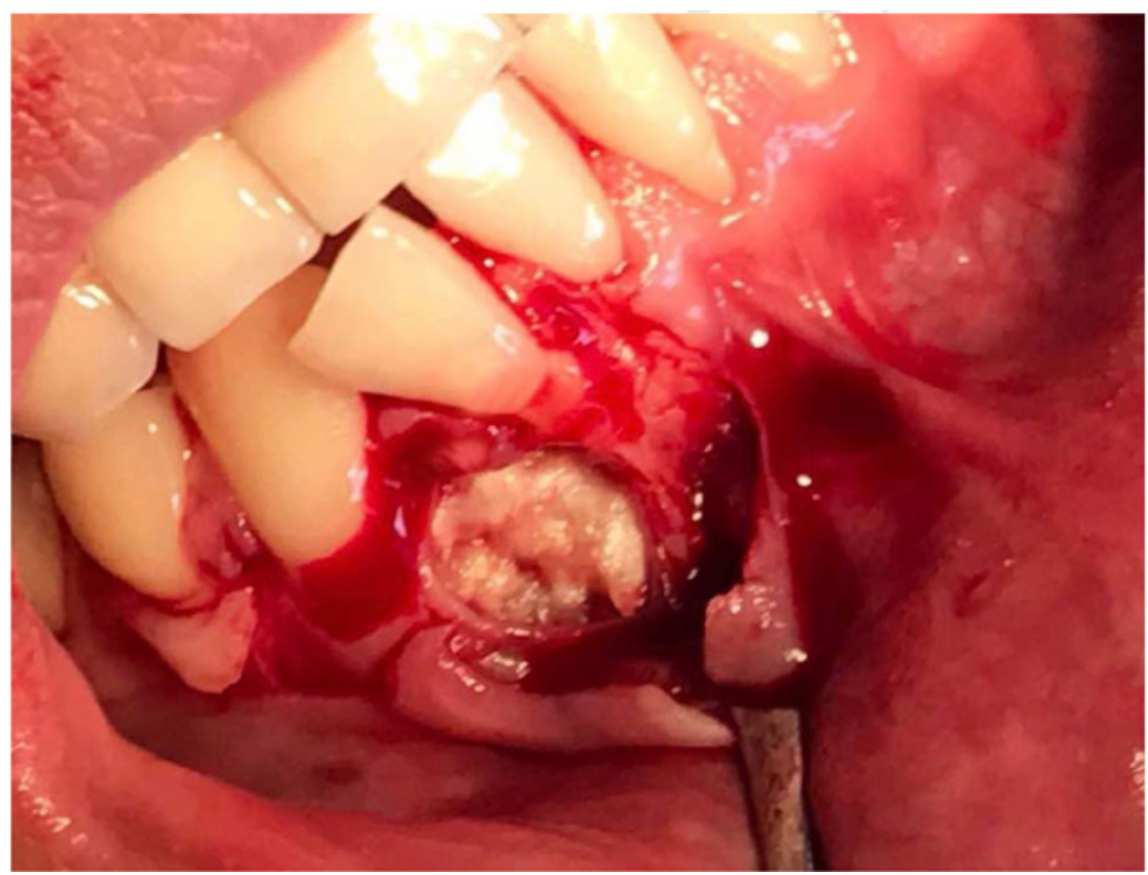

Figura 4. Acto quirúrgico: exposición de la lesión.

Fuente: elaboración propia

La cápsula del quiste se desprendió del hueso adyacente y se procedió a la enucleación total de la lesión tomándola con una pinza forcé-presión, ejerciendo una leve tensión y separando con una cureta quirúrgica la adherencia al tejido óseo. Luego de haber realizado la enucleación de la lesión se procedió a curetear el hueso circundante buscando zonas de clivaje y posibles restos remanentes posterior a la enucleación (figura 5). 


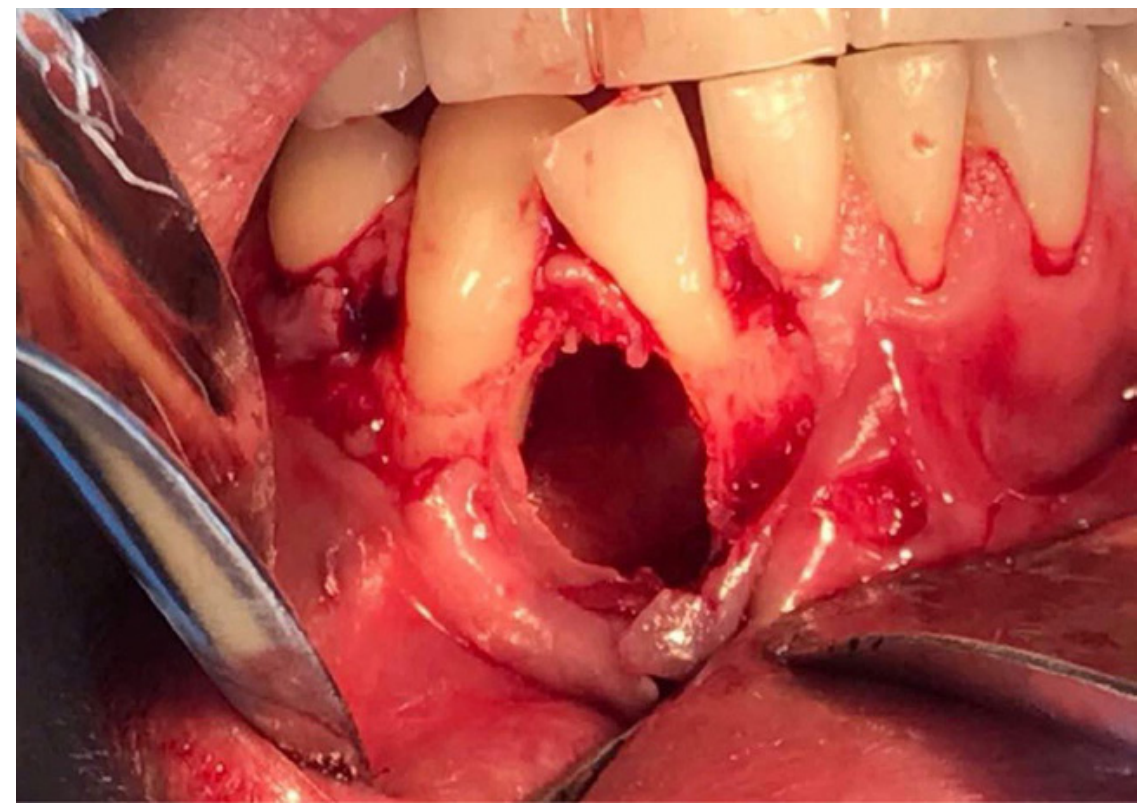

Figura 5. Acto quirúrgico. Cavidad ósea posterior a la enucleación de la lesión quística. Fuente: elaboración propia

Posteriormente se tomó biopsia excisional de la lesión y se envió a examinar histológicamente, arrojó como diagnóstico definitivo quiste periodontal lateral (figuras 6 y 7 ).

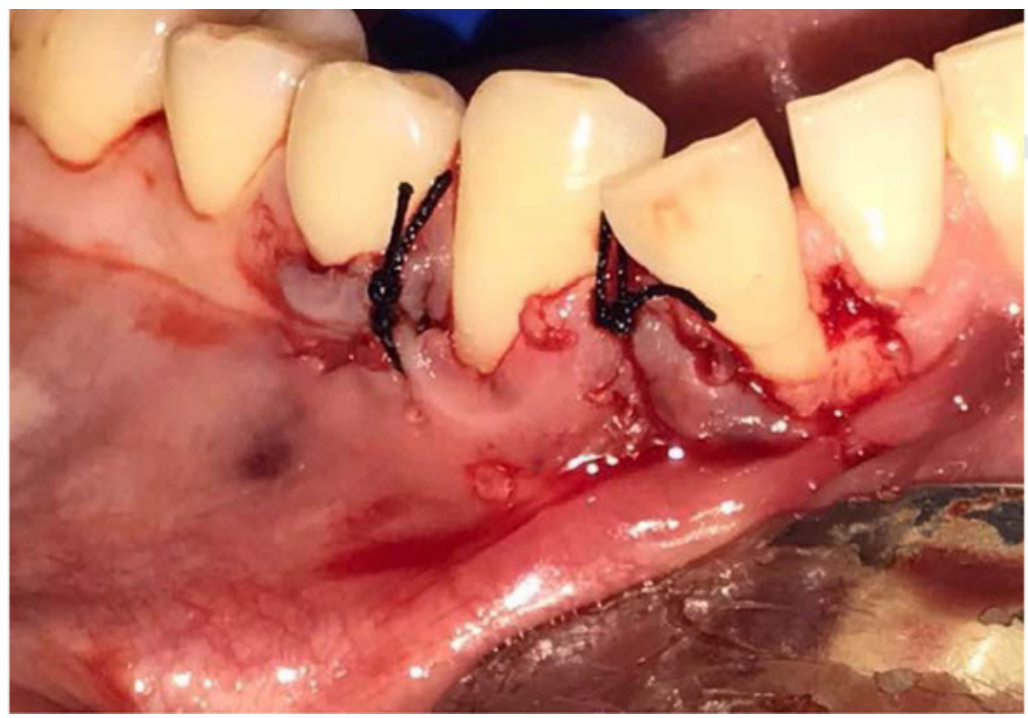

Figura 6. Sutura.

Fuente: elaboración propia 


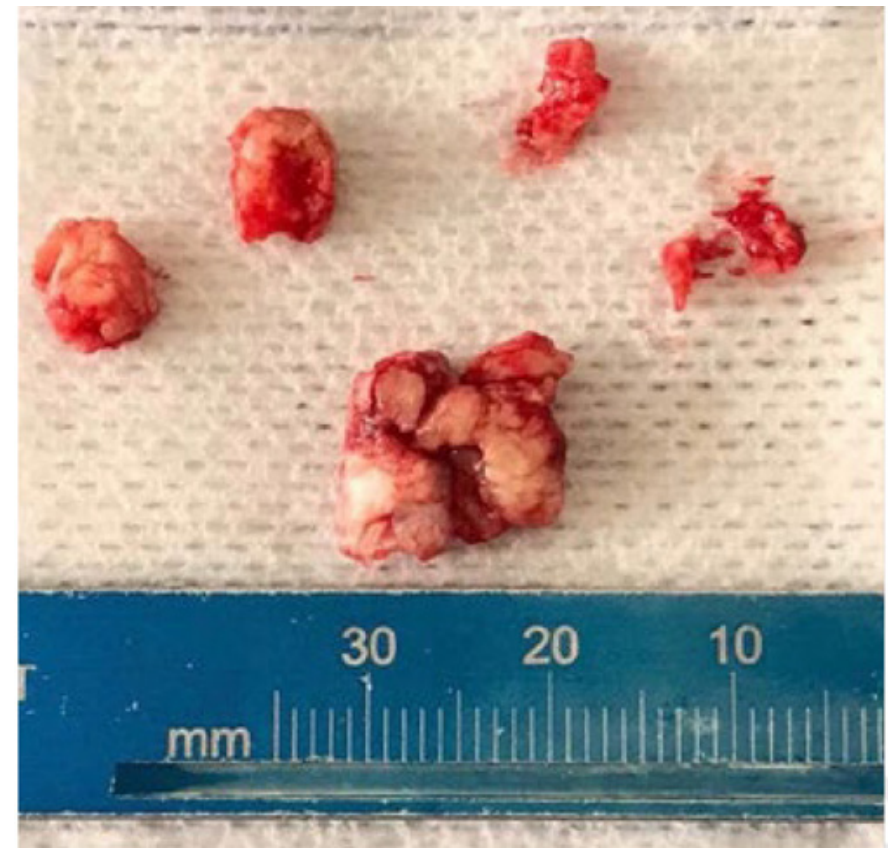

Figura 7. Pieza Macroscópica

Fuente: Elaboración propia

\section{Discusión}

La literatura establece como diagnósticos diferenciales del QPL, el queratoquiste odontogénico, que presenta elevada agresividad y alto potencial de recurrencia después del tratamiento. El quiste radicular, que aparece con el diente no vital (9); ya que ninguno de los hallazgos anteriores existe en el presente caso, se diagnostica como QPL en base a la radiografía clínica y reconfirmado histológicamente.

Según reportes, el QPL se presenta con frecuencia en individuos entre la quinta y séptima década de la vida, sin embargo, se observa en el caso descrito que puede presentarse la tercera década.

Se informó que el QPL no tiene predilección por ninguna raza y aunque algunos estudios reportan la distribución de igual sexo, otros informan la preponderancia masculina $(4,17)$.

Radiográficamente, la literatura reporta que el QPL muestra un área radiolúcida redonda u ovoide circunscrita y la mayoría posee menos de $1 \mathrm{~cm}$ de diámetro, lo cual contrasta con el caso actual que presenta $1 \mathrm{~cm}$ de diámetro siendo mayor. 


\section{Conclusiones}

La enucleación de la lesión en un solo tiempo quirúrgico y la posterior evaluación histológica para confirmar el diagnóstico, constituye un tratamiento satisfactorio para este tipo de patologías. La recurrencia es infrecuente, sin embargo, es necesario mantener un monitoreo radiográfico del sitio de la lesión.

La importancia y el objetivo de ésta investigación y presentación de caso es hacer énfasis en el diagnóstico precoz y tratamiento temprano a través del estudio clínico, radiográfico y microscópico, en la prevención de su desarrollo y la consecuente pérdida del tejido óseo.

\section{Referencias}

1. El-Naggar AK, Chan JKC, Grandis JR, Takata T, Slootweg PJ. WHO Classification of Head and Neck Tumours. Lyon: International Agency for Research on Cancer; 2017, 204-60 p.

2. Philipsen HP, Reichart PA. Classification of odontogenic tumours. A historical review. J Oral Pathol Med. 2006; 35(9): 525-9. doi: 10.1111/j.1600-0714.2006.00470.x

3. Das Chagas LF, Faing C, Guimarães LA, Aigotti A, Brandão H, Dias J. Periodontal Cyst: a Case Report and Literature Review. J Oral Maxillofac Res. 2010; 1(4): e5. doi: 10.5037/jomr.2010.1405

4. Wood K, Goaz P. Differential Diagnosis of Oral and Maxillofacial Lesions. St. Louis: Mosby; 1997.

5. Demirkol M, Ege B, Yanik S, Aras MH, Ay S. Clinicopathological study of jaw cysts in southeast region of Turkey. Eur J Dent. 2014; (8): 107-11. doi: 10.4103/1305-7456.126260

6. Adamala SR, Talla H, Medikonda SK, Soujanya S. Lateral periodontal cyst: An outlandish anamnesis. J Indian Acad Oral Med Radiol. 2014; (26): 351-4. doi:10.4103/0972-1363.145031

7. Formoso Senande MF, Figueiredo R, Berini Aytés L, Gay Escoda C. Lateral periodontal cysts: A retrospective study of 11 cases. Med Oral Patol Oral Cir Bucal. 2008; 13: E313-7.

8. Ortega A, Fariña V, Gallardo A, Espinoza I, Acosta S. Nonendodontic periapical lesions: a retrospective study in Chile. Int Endod J. 2007; 40(5): 386-90. doi: 10.1111/j.1365-2591.2007.01232.x 
9. Nikitakis NG, Brooks JK, Melakopoulos I, Younis RH, Scheper MA, Pitts MA, Al-Mubarak H, Sklavounou A. Lateral periodontal cysts arising in periapical sites: a report of two cases. J Endod. 2010; 36(10): 1707-11. doi: 10.1016/j.joen.2010.06.015

10. Cohen DA, Neville BW, Damm DD, White DK. The lateral periodontal cyst. A report of 37 cases. J Periodontol. 1984; 55(4): 230-4. doi: 10.1902/jop.1984.55.4.230

11. Altini M, Shear M. The lateral periodontal cyst: An update. J Oral Pathol Med. 1992; (21): 24550. doi: 10.1111/j.1600-0714.1992.tb01004.x

12. Kramer IR, Pindborg JJ, Shear M. WHO Histological Typing of Odontogenic Tumours. Geneva: Springer-Verlag; 1992, 34-118 p.

13. Angelopoulou E, Angelopoulos AP. Lateral periodontal cyst. Review of the literature and report of a case. J Periodontol. 1990; (61): 126-31. doi: 10.1902/jop.1990.61.2.126

14. Demirkol M, Ege B, Yanik S, Aras MH, Ay S. Clinicopathological study of jaw cysts in southeast region of Turkey. Eur J Dent. 2014; (8): 107-11. doi: 10.4103/1305-7456.126260

15. Nart J, Gagari E, Kahn MA, Griffin TJ. Use of guided tissue regeneration in the treatment of a lateral periodontal cyst with a 7-month reentry. J Periodontol. 2007; (78): 1360-4. doi: 10.1902/ jop.2007.060483

16. Subramaniam P, Kumar K, Ramakrishna T, Bhadranna A. Bone regeneration with plasma-rich- protein following enucleation of traumatic bone cyst. Eur J Dent. 2013; (7): 377-81. doi: 10.4103/1305-7456.115427

17. Suleyman Emre Meseli, Omer Birkan Agrali, Onder Peker, and Leyla Kuru. Treatment of lateral periodontal cyst with guided tissue regeneration. Eur J Dent. 2014; 8(3): 419-423. doi: 10.4103/1305-7456.137661 